\title{
EFEITOS DE NÍVEIS DE CÁLCIO COMBINADOS COM DIFERENTES CONCENTRAÇÕES DE SAIS NA TOLERÂNCIA DE TRIGO À TOXICIDADE DE ALUMÍNIO, EM SOLUÇÃO NUTRITIVA ( ${ }^{(1)}$
}

\author{
CARLOS EDUARDO DE OLIVEIRA CAMARGO $\left({ }^{2}\right)$
}

\begin{abstract}
RESUMO
Com o objetivo de estudar o efeito do cálcio na tolerância à toxicidade de alumínio, foi instalado um experimento empregando três cultivares de trigo - BH1146, IAC-17 e Siete Cerros - em soluçōes nutritivas contendo $10 \mathrm{mg} /$ litro de $\mathrm{Al}^{3+}$ combinados com três concentraf̧̋es de sais - um décimo, um quinto e metade da concentração de sais da soluçâao nutritiva completa - e quatro níveis de cálcio - 16, 80,160 e $320 \mathrm{mg} /$ litro. A tolerância foi medida pela capacidade de as raízes primárias continuarem a crescer em soluções sem ahumínio após a permanência de 24,48 e 72 horas em soluçōes de tratamento contendo determinada concentraçāo de sais combinada com determinada concentraçăo de cálcio na presença de $10 \mathrm{mg} /$ litro de $\mathrm{Al}^{3+}$. Independente da concentração salina da solução, quando foi empregada a concentração de $16 \mathrm{mg} /$ litro de cálcio, todos os cultivares foram tolerantes quando o tempo de permanência na solução com $\mathrm{Al}^{3}+$ foi de 24 horas; e os cultivares Bh-1146 e IAC-17 foram tolerantes e Siete Cerros, sensível, quando o tempo de permanência na solução com $\mathrm{Al}^{3+}$ foi de 48 e 72 horas. $\mathrm{Na}$ concentração de $80 \mathrm{mg} /$ litro de cálcio, verificou-se o mesmo resultado; entretanto, 'Siete Cerros' mostrou-se tolerante na solução salina mais concentrada (metade da concentração da solução completa) quando o tempo de permanência na soluçäo com $10 \mathrm{mg} / \mathrm{litro}$ de $\mathrm{Al}^{3+}$ foi de 48 e 72 horas. Nas soluçoes com 160 e $320 \mathrm{mg} /$ litro, todos os cultivares em estudo apresentaram tolerância, independente do tempo de permanência nas soluções e das concentrações de sais utilizadas. O sintoma de toxicidade de $\mathrm{Al}^{3+}$ ficou acentuado pela diminuição da con-
\end{abstract}

$\left(^{1}\right)$ Trabalho apresentado na XIII Reuniāo Nacional de Pesquisa de Trigo, Cruz Alta (RS), 1984. Com verba suplementar do Acordo do Trigo entre as Cooperativas de Produtores Rurais do Vale do Paranapanema e a Secretaria de Agricultura e Abastecimento, por meio do Instituto Agronômico. Recebido para publicaçđ̃o em 10 de agosto de 1984.

( ${ }^{2}$ Seçāo de Arroz e Cereais de Invemo, Instituto Agronômico, Caixa Postal 28, 13100 Campinas, SP. Com bolsa de suplementação do CNPq. 
centração de cálcio e/ou da concentração salina e pelo aumento no período de permanência na solução de $\mathrm{Al}^{3+}$ para os três cultivares estudados.

Termos de indexação: trigo; toxicidade; alumínio; concentração de sais; níveis de cálcio; solução nutritiva; raízes primárias; comprimento da raiz.

\section{INTRODUÇÃO}

O efeito primário da toxicidade de alumínio no trigo é, segundo KERRIDGE : (1969), a paralisação irreversivel do crescimento da raiz devida a uma inibição da elongação das células.

O controle do complexo mineral do solo ê bastante difícil para que determinado nível de alumínio possa ser reproduzido de um experimento para outro. $O$ emprego de soluções nutritivas pode tornar mais eficiente e precisa a separação das plantas em relação à tolerância ao alumínio, conforme CAMPBELL \& LAFEVER (1976).

Os resultados obtidos por CAMARGO \& OLIVEIRA (1981) medindo os comprimentos das raízes de dez cultivares de trigo após 72 horas de crescimento em solução nutritiva completa, com desenvolvimento prévio, por 48 horas, em soluções de tratamentos com um décimo de concentração salina da solução normal contendo cinco diferentes níveis de $\mathrm{Al}^{3}{ }^{+}$, permitiram a separação desses cultivares em cinco classes de tolerância. No mesmo trabalho, estudando os cultivares de trigo Tobari-66, Alondra-S-46, IAC-17, BH-1146 e Siete Cerros em solos ácidos com a presença de $\mathrm{Al}^{3+}$ esses autores confirmaram a possibilidade de separar classes de tolerância a tais condições.

As raízes dos cultivares de trigo BH-1146, IAC-17 e Siete Cerros não cresceram após 72 horas em contacto com uma soluçąo nutritiva completa, quando anteriormente cultivados por 48 horas em solução nutritiva de tratamento com um décimo da concentração de sais da solução completa e com ausência de cálcio e alumínio. Nessa condição, a ausência de cálcio paralisou irreversivelmente o crescimento das raízes primárias dos três cultivares. Entretanto, quando o mesmo procedimento foi executado, utilizando, porém, as soluções nutritivas de tratamento com concentração de sais de um quinto e metade da solução completa, verificou-se um crescimento médio superior a $20 \mathrm{~mm}\left(^{3}\right)$.

(3) CAMARGo, C.E.O. Efeito da ausên cia do cálcio em solução nutritiva no crescimento das raízes de cultivares de trigo. Seção de Arroz e Cereais de Inverno, Instituto Agronômico. (Não publicado) 
OUELLETTE \& DESSUREAUX (1958) postularam que um dos efeitos do cálcio era diminuir a absorção de alumínio pelas plantas: a maior necessidade de cálcio na presença de alumínio para manter o crescimento da planta poderia ser devida a um efeito de o cálcio decrescer a absorção do alumínio. CLARKSON \& SANDERSON (1971) e OUELLETTE \& DESSUREAUX (1958) afirmaram que uma quantidade maior de cálcio era requerida para manter um crescimento normal das plantas em presença do alumínio.

Para CLARKSON \& SANDERSON (1971), a inibição do crescimento radicular causada pelo alumínio poderia ser parcialmente vencida se a concentração de cálcio no meio fosse aumentada; entretanto, o efeito inibidor do alumínio ainda estaria presente. Estudos posteriores demonstraram que raizes tratadas com alumínio transportavam muito menos cálcio para as partes aéreas.

O presente trabalho teve por objetivo estudar o comportamento de três cultivares de trigo em relação à tolerância à toxicidade de alumínio, quando submetidos a diferentes períodos de crescimento em soluções de tratamento contendo quatro niveis de cálcio combinados com três concentrações salinas.

\section{MATERIAL E MÉTODOS}

O delineamento estatístico empregado foi de parcelas subsubdivididas com duas repetições: as parcelas foram compostas por 12 diferentes soluções de tratamento; as subparcelas, pelo tempo de crescimento das plântulas nas soluções de tratamento (24, 48 e 72 horas), e as subsubparcelas, pelos três cultivares de trigo (BH-1 146, IAC-17 e Siete Cerros). Os dados foram analisados, considerando-se a média de crescimento da raiz primária central das 20 plântulas de cada cultivar após a influência prejudicial do $\mathrm{Al}^{3+}$ nas soluções de tratamento.

O método utilizado consistiu no seguinte: as sementes dos três cultivares foram cuidadosamente lavadas com uma solução de hipoclorito de sódio a $10 \%$ e colocadas para germinar em caixas de Petri por 24 horas. Após este tempo, as radículas estavam iniciando a emergência. Em cada repetição, foram utilizadas 36 telas de náilon, contendo cada uma as 20 sementes uniformes de cada um dos cultivares em estudo. Cada uma das telas foi colocada em contato com a solução nutritiva completa existente em 36 vasilhas plásticas de 8,3 litros de capacidade. A composição da solução nutritiva completa era a seguinte: $\mathrm{Ca}\left(\mathrm{NO}_{3}\right)_{2} 4 \mathrm{mM} ; \mathrm{MgSO}_{4} 2 \mathrm{mM} ; \mathrm{KNO}_{3} 4 \mathrm{mM}$; $\left(\mathrm{NH}_{4}\right)_{2} \mathrm{SO}_{4} 0,435 \mathrm{mM} ; \mathrm{KH}_{2} \mathrm{PO}_{4} 0,5 \mathrm{mM} ; \mathrm{MnSO}_{4} 2 \mu \mathrm{M} ; \mathrm{CuSO}_{4} 0,3 \mu \mathrm{M} ; \mathrm{ZnSO}_{4}$ $0,8 \mu \mathrm{M} ; \mathrm{NaCl} 30 \mu \mathrm{M} ; \mathrm{Fe}-\mathrm{CYDTA} 10 \mu \mathrm{M} ; \mathrm{Na}_{2} \mathrm{MoO}_{4} 0,1 \mu \mathrm{M}$ e $\mathrm{H}_{3} \mathrm{BO}_{3} 10 \mu \mathrm{M}$. 
O nível da solução nas vasilhas plásticas tocou a parte de baixo da tela de náilon, de modo que as sementes foram mantidas úmidas e as radículas emergentes tinham um pronto suprimento de nutrientes. $\mathrm{O} \mathrm{pH}$ da solução foi previamente ajustado para $4,0 \mathrm{com}$ uma solução de $\mathrm{H}_{2} \mathrm{SO}_{4} 1 \mathrm{~N}$, e as vasilhas plásticas contendo as soluçoes, colocadas em banho-maria com temperatura de $25 \pm 1^{\circ} \mathrm{C}$ dentro do laboratório.

Após desenvolver-se nessas condições por 48 horas, cada plântula tinha três raízes primárias, uma mais longa e duas mais curtas localizadas lateralmente à primeira.

As 36 telas contendo as 20 plântulas de cada cultivar foram divididas em três grupos de 12. Cada grupo foi colocado em contato com 12 soluções de tratamento contendo $10 \mathrm{mg} /$ litro de $\mathrm{Al}^{3+}$ combinado com diferentes níveis de cálcio e concentração salina, conforme a relação seguinte:

1. Um décimo da concentração de sais da solução nutritiva completa e $16 \mathrm{mg} /$ litro de Ca. (Este tratamento é usado como rotina para seleção visando à tolerância ao $\mathrm{Al}^{3+}$ em trigo no Instituto Agronômico.)

2. Um décimo da concentração de sais da solução nutritiva completa e $80 \mathrm{mg} /$ litro de Ca.

3. Um décimo da concentração de sais da solução nutritiva completa e $160 \mathrm{mg} /$ litro de $\mathrm{Ca}$

4. Um décimo da concentração de sais da solução nutritiva completa e $320 \mathrm{mg} /$ litro de Ca.

5. Um quinto da concentração de sais da solução nutritiva completa e $16 \mathrm{mg} /$ litro de $\mathrm{Ca}$.

6. Um quinto da concentração de sais da solução nutritiva completa e $80 \mathrm{mg} /$ litro de $\mathrm{Ca}$.

7. Um quinto da concentração de sais da solução nutritiva completa e $160 \mathrm{mg} /$ litro de Ca.

8. Um quinto da concentração de sais da solução nutritiva completa e $320 \mathrm{mg} /$ litro de Ca.

9. Metade da concentração de sais da solução nutritiva completa e $16 \mathrm{mg} /$ litro de $\mathrm{Ca}$.

10. Metade da concentração de sais da solução nutritiva completa e $80 \mathrm{mg} /$ litro de Ca.

11. Metade da concentração de sais da solução nutritiva completa e $160 \mathrm{mg} /$ litro de Ca.

12. Metade da concentração de sais da solução nutritiva completa e $320 \mathrm{mg} /$ litro de Ca. 
No primeiro grupo, as plântulas cresceram nas soluções de tratamento durante 24 horas; no segundo, durante 48 horas e no terceiro, durante 72 horas.

Nas soluções de tratamento, o fósforo foi omitido para evitar a possível precipitação do alumínio, e o ferro foi adicionado em quantidade equivalente àquela colocada na solução completa como $\mathrm{FeCl}_{3}$ no lugar do $\mathrm{Fe}-$ CYDTA, como descrito por ALI (1973) e MOORE et alii (1976). Antes da transferência das telas contendo as plântulas para as soluções de tratamento, adicionou-se a quantidade necessária de $\mathrm{Ca}\left(\mathrm{NO}_{3}\right)_{2}$ e $\mathrm{Al}_{2}\left(\mathrm{SO}_{4}\right)_{3}$, de modo a serem obtidas as concentrações desejadas de cálcio e alumínio, suficiente solução de $\mathrm{H}_{2} \mathrm{SO}_{4} 1 \mathrm{~N}$ para ser obtido $\mathrm{pH} 4,0$.

As plantas permaneceram crescendo por 24,48 e 72 horas nas soluções de tratamento em banho-maria a $25 \pm 1^{\circ} \mathrm{C}$. Decorridos esses períodos, as telas foram transferidas de volta para as 36 vasilhas plásticas contendo as soluçōes nutritivas completas, onde cresceram antes das soluções de tratamento, por mais 72 horas com temperatura de $25 \pm 1^{\circ} \mathrm{C}$. O crescimento das raízes primárias nessas 72 horas depende da severidade do prévio tratamento; nos mais severos, elas não crescem mais e permanecem grossas, mostrando no ápice uma injúria típica com descoloramento. $O$ crescimento da raiz de cada plântula foi determinado, medindo-se o comprimento da raiz primária central no final de 72 horas de crescimento na solução nutritiva completa, e subtraindo-se do seu comprimento no final do crescimento na solução de tratamento.

Durante todo o experimento, o $\mathrm{pH}$ das soluções nutritivas completas e de tratamento foi mantido o mais próximo possível de 4,0 , por ajustamentos diários com uma solução de $\mathrm{H}_{2} \mathrm{SO}_{4} 1 \mathrm{~N}$, e empregada luz fluorescente e arejamento das soluçōes.

\section{RESULTADOS E DISCUSSÃO}

O comprimento médio das raízes dos cultivares BH-1 146, IAC-17 e Siete Cerros, após 72 horas de crescimento na solução nutritiva completa, seguido de 24 horas de crescimento nas soluções de tratamento contendo quatro concentrações de cálcio combinadas com três concentrações salinas, encontram-se no quadro 1.

Quando foram adicionados nas soluções de tratamento $16,80,160$ e $320 \mathrm{mg} /$ litro de $\mathrm{Ca}$, verificou-se, independente da concentração salina utilizada, que todos os cultivares estudados mostraram reação de tolerância, exibindo crescimento radicular nas soluções nutritivas completas após crescimento por 24 horas nas soluções de tratamento. 
QUADRO 1 - Comprimento médio das raízes de três cultivares de trigo medidos após 72 horas de crescimento nas soluçб̄es nutritivas completas seguido de crescimento de 24 horas nas soluçōes contendo $10 \mathrm{mg} /$ litro de $\mathrm{Al}^{3+}$ com quatro concentraçðes de cálcio combinadas com três diferentes concentraçðes salinas

\begin{tabular}{lccccc}
\hline \multirow{2}{*}{ Cultivares } & $\begin{array}{c}\text { Concentração } \\
\text { salina } \\
\text { (força iônica } \\
\text { da solução) }\end{array}$ & \multicolumn{4}{c}{$\begin{array}{c}\text { Concentração de cálcio nas soluçðes nutritivas } \\
\text { (mg/litro) }\end{array}$} \\
\cline { 3 - 6 } & & 16 & 80 & 160 & 320 \\
\cline { 3 - 5 } BH-1146 & $1 / 10$ & 48,7 & 67,3 & 103,4 & 106,4 \\
& $1 / 5$ & 50,4 & 75,0 & 97,5 & 109,4 \\
& $1 / 2$ & 55,1 & 93,2 & 94,1 & 95,1 \\
IAC-17 & $1 / 10$ & 33,2 & 47,4 & 76,1 & 99,8 \\
& $1 / 5$ & 38,1 & 56,7 & 89,2 & 109,3 \\
& $1 / 2$ & 41,7 & 84,3 & 102,9 & 103,4 \\
Siete Cerros & $1 / 10$ & & & & \\
& $1 / 5$ & 0,5 & 23,7 & 45,9 & 44,6 \\
& $1 / 2$ & 2,8 & 27,0 & 48,6 & 59,4 \\
& 14,0 & 50,5 & 54,8 & 47,3 \\
\hline
\end{tabular}

Para a concentração de 16 e $80 \mathrm{mg} /$ litro, verificou-se para os três cultivares um aumento no crescimento das raízes primárias, à medida que se aumentaram as concentraçðes salinas, nas soluçðes de tratamento, de um décimo para metade da concentração empregada na solução nutritiva completa. O mesmo se observou para os cultivares IAC-17 e Siete Cerros, quando se empregaram $160 \mathrm{mg} /$ litro de $\mathrm{Ca}$ nas soluções de tratamento. Tais resultados estão de acordo com os obtidos por CAMARGO et alii (1981) e CAMARGO (1984), que mostraram que a tolerância à toxicidade a determinada concentração de $\mathrm{Al}^{3}+$ é uma característica antes relativa do que absoluta, por depender da concentração de sais presentes na-solução. Aumentando-se a concentração salina na solução, diminuiu o efeito inibitório do alumínio devido à redução de sua a tividade ionnica.

O comprimento médio das raízes dos três cultivares, medidos após 72 horas de crescimento na solução nutritiva completa seguido de 48 e 72 horas de cultivo nas soluções de tratamento contendo quatro concentrações de Ca combinadas com três concentrações salinas encontram-se nos quadros 2 e 3 respectivamente. 
QUADRO 2 - Comprimento médio das ráizes de três cultivares de trigo medidos após 72 horas de crescimento nas soluçðes nutritivas completas seguido de crescimento de 48 horas nas soluçðes contendo $10 \mathrm{mg} /$ litro de $\mathrm{Al}^{3+}$ com quatro concentraçóes de cálcio combinadas com três diferentes concentraçðes salinas

\begin{tabular}{|c|c|c|c|c|c|}
\hline \multirow{2}{*}{ Cul tivares } & \multirow{2}{*}{$\begin{array}{c}\text { Concentração } \\
\text { salina } \\
\text { (força iônica } \\
\text { da soluçăo) }\end{array}$} & \multicolumn{4}{|c|}{$\begin{array}{l}\text { Concentração de cálcio nas soluçōes nutritivas } \\
\text { (mg/litro) }\end{array}$} \\
\hline & & 16 & 80 & 160 & 320 \\
\hline & & & & & 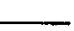 \\
\hline \multirow[t]{3}{*}{ BH-1 146} & $1 / 10$ & 30,3 & 71,1 & 92,8 & 112,2 \\
\hline & $1 / 5$ & 32,6 & 72,1 & 100,5 & 105,7 \\
\hline & $1 / 2$ & 44,4 & 90,8 & 101,8 & 107,9 \\
\hline \multirow[t]{3}{*}{ IAC -17} & $1 / 10$ & 5,2 & 49,1 & 83.3 & 103,7 \\
\hline & $1 / 5$ & 8,8 & 55,9 & 76,6 & 107,9 \\
\hline & $1 / 2$ & 35,5 & 60,0 & 81,4 & 105,3 \\
\hline \multirow[t]{3}{*}{ Siete Cerros } & $1 / 10$ & 0,0 & 0,0 & 13,1 & 21,1 \\
\hline & $1 / 5$ & 0,0 & 0,0 & 22,0 & 19,8 \\
\hline & $1 / 2$ & 0,0 & 19,9 & 18,4 & 24,7 \\
\hline
\end{tabular}

QUADRO 3 - Comprimento médio das rałzes de três cultivares de trigo medidos após 72 horas de crescimento nas soluçðes nutritivas completas, seguido de crescimento de 72 horas nas soluçðes contendo $10 \mathrm{mg} /$ litro de $\mathrm{Al}^{3+}$ com quatro concentraçð̄es de cálcio combinadas com três diferentes concentraçóes salinas

\begin{tabular}{|c|c|c|c|c|c|}
\hline \multirow{2}{*}{ Cultivares } & \multirow{2}{*}{$\begin{array}{c}\text { Concentração } \\
\text { salina } \\
\text { (força iônica } \\
\text { da solução) }\end{array}$} & \multicolumn{4}{|c|}{$\begin{array}{l}\text { Concentraçăo de cálcio nas soluçðes nutritivas } \\
\text { (mg/litro) }\end{array}$} \\
\hline & & 16 & 80 & 160 & 320 \\
\hline & & & & $\ldots$ & - \\
\hline \multirow[t]{3}{*}{ BH-1 146} & $1 / 10$ & 25,4 & 81,1 & 116,8 & 130,6 \\
\hline & $1 / 5$ & 34,6 & 102,9 & 112,1 & 133,2 \\
\hline & $1 / 2$ & 54,8 & 84,2 & 113,6 & 118,3 \\
\hline \multirow[t]{3}{*}{ IAC-17 } & $1 / 10$ & 9,6 & 55,9 & 94,4 & 118,0 \\
\hline & $1 / 5$ & 11,6 & 63,9 & 93,6 & 139,1 \\
\hline & $1 / 2$ & 30,0 & 87,9 & 123,2 & 117,2 \\
\hline \multirow[t]{3}{*}{ Siete Cerros } & $1 / 10$ & 0,0 & 0,0 & 3,1 & 21,2 \\
\hline & $1 / 5$ & 0,0 & 0,0 & 1,7 & 16,5 \\
\hline & $1 / 2$ & 0,0 & 3,5 & 10,5 & 14,1 \\
\hline
\end{tabular}

Quando se adicionaram 16, 80, 160 e $320 \mathrm{mg} /$ litro de Ca na presença de qualquer uma das concentrações salinas consideradas e independendo do tempo de crescimento nas soluções de tratamento (48 ou 72 horas), os cultivares BH-1146 e IAC-17 foram tolerantes a $10 \mathrm{mg} /$ litro de $\mathrm{Al}^{3+}$. Siete 
Cerros mostrou-se sensivel, apresentando inibição irreversível do crescimento radicular, quando foi cultivado durante 48 e 72 horas em soluções de tratamento contendo $10 \mathrm{mg} /$ litro de $\mathrm{Al}^{3+}$ e com um décimo, um quinto e metade da concentração salina da solução nutritiva completa com a presença de $16 \mathrm{mg} /$ litro de $\mathrm{Ca}$. Este cultivar foi sensível quando crescido durante 48 e 72 horas nas soluções de tratamento com um décimo e um quinto da concentração salina e com a adição de $80 \mathrm{mg} /$ litro de Ca, porém mostrou-se tolerante a $10 \mathrm{mg} /$ litro de $\mathrm{Al}^{3+}$ quando se empregou esta mesma concentração de $\mathrm{Ca}$ em combinação com metade da concentração salina da solução completa, independente do tempo de crescimento (48 ou 72 horas). Foi ainda tolerante ao $\mathrm{Al}^{3+}$ quando se acrescentaram 160 e $320 \mathrm{mg} /$ litro de $\mathrm{Ca}$ nas soluções de tratamento, independente da concentração salina e do tempo de crescimento. Estes resultados confirmaram que seria necessária maior quantidade de cálcio na presença de $\mathrm{Al}^{3+}$ para manter o crescimento radicular (OUELLETTE \& DESSUREAUX, 1958).

Nas soluções de tratamento contendo 16 e $80 \mathrm{mg} /$ litro de $\mathrm{Ca}$, aumentando-se o tempo de permanência de 24 para 72 horas, para as três concentrações salinas utilizadas, os efeitos prejudiciais do $\mathrm{Al}^{3+}$, entre eles a diminuição do crescimento do sistema radicular, foram mais acentuados para os três cultivares.

O aumento da concentração salina nas soluções de tratamento contendo 16 e $80 \mathrm{mg} /$ litro de $\mathrm{Ca}$, independendo do tempo de desenvolvimento das plantas nessas condiçōes, mostrou um efeito menos acentuado da toxicidade de $\mathrm{Al}^{3+}$; portanto, as plantas dos três cultivares apresentaram-se com maior crescimento radicular.

$O$ efeito da concentração salina (força iônica da solução) e do tempo de crescimento nas soluções de tratamento, quando se colocaram 160 ou $320 \mathrm{mg} /$ litro de $\mathrm{Ca}$, foi pouco evidente em virtude de a elevada concentração de Ca diminuir a absorção do alumínio (CLARKSON \& SANDERSON, 1971).

\section{CONCLUSÕES}

1. O cultivar BH-1146 foi tolerante; 'IAC-17', moderadamente tolerante, e 'Siete Cerros', sensível a $10 \mathrm{mg} /$ litro de $\mathrm{Al}^{3+}$, quando se empregaram soluções com $16 \mathrm{mg} /$ litro de $\mathrm{Ca}$, independendo do tempo de crescimento e das concentrações salinas das soluções de tratamento.

2. O sintoma de toxicidade de $\mathrm{Al}^{3+}$ ficou acentuado pela diminuição da concentração de cálcio e/ou da concentração salina e pelo aumento no período de crescimento na solução contendo $10 \mathrm{mg} /$ litro de $\mathrm{Al}^{3+}$, para os três cultivares. 


\title{
SUMMARY \\ INFLUENCE OF CALCIUM LEVELS COMBINED WITH SALT CONCENTRATIONS IN NUTRIENT SOLUTION TO ALUMINUM TOXICITY IN WHEAT CULTIVARS
}

\begin{abstract}
An experiment was conducted to study the cultivars BH-1146, IAC-17 and Siete Cerros in nutrient solutions containing $10 \mathrm{mg} / 1$ of $\mathrm{Al}^{3+}$ combined with three salt concentrations (one tenth, one fifth and a half of the salt concentration of the complete nutrient solution) and four levels of $\mathrm{Ca}(16,80,160$ and $320 \mathrm{mg} / \mathrm{l})$. The tolerance was evaluated by measuring the root growth in an aluminum-free complete nutrient solution after a treatment of 24,48 and 72 hours in treatment solutions containing a particular salt concentration combined with a particular level of $\mathrm{Ca}$ in the presence of $10 \mathrm{mg} / 1$ of $\mathrm{Al}^{3+}$. Independently of the salt concentration, the level of $16 \mathrm{mg} / 1$ of $\mathrm{Ca}$ into the solution showed all wheat cultivars under study as tolerant when the growth period into the treatment solutions was 24 hours; and 'BH-1146', and 'IAC-1 7' showed tolerance and 'Siete Cerros' sensitivity to $10 \mathrm{mg} / 1$ of $\mathrm{Al}^{3+}$ when the growth period into the treatment solution was 48 and 72 hours. When it was used $80 \mathrm{mg} / 1$ of $\mathrm{Ca}$ into the treatment solution the same results were obtained, but 'Siete Cerros' presented $\mathrm{Al}$ tolerance when it was used treatment solutions with a half of the salt concentration of the complete nutrient solution and the growth period in this solution was 48 and 72 hours. All studied cultivars presented tolerance to $\mathrm{Al}$ when it was placed into the treatment solutions 160 and $320 \mathrm{mg} / 1 \mathrm{of} \mathrm{Ca}$, independently of the growth period and of the salt concentration. Aluminum toxicity symptom was increased to all studied cultivars by decreasing the Ca concentration and/or the salt concentration and by increasing the growth period in a treatment solution with $10 \mathrm{mg} / 1$ of $\mathrm{Al}^{3+}$.
\end{abstract}

Index terms: wheat; aluminum toxicity; salt concentration; calcium levels; nutrient solution; primary roots; root lenght.

\section{REFERÊNCIAS BIBLIOGRÁFICAS}

ALI, S.M.E. Influence of cations, on aluminum toxicity in wheat (Triticum aestivum Vill., Host). Corvallis, Oregon State University, 1973. $102 \mathrm{f}$.

CAMARGO, C.E.O. Tolerância de cultivares de arroz a dois níveis de alumínio em soluções nutritivas contendo diferentes concentrações de sais. Bragantia, Campinas, 43(2):381-388, 1984.

\& OLIVEIRA, O.F. Tolerância de cultivares de trigo a diferentes níveis de alumínio em solução nutritiva e no solo. Bragantia, Campinas, 40: $21-31,1981$.

\& LAVORENTI, A. Efeito de diferentes concentrações de sais em solução nutritiva na tolerância de cultivares de trigo à toxicidade de alumínio. Bragantia, Campinas, 40:93-101, 1981.

CAMPBELE, L.G. \& LAFEVER, H.N. Correlation of field and nutrient culture techniques of screening wheat for aluminum tolerance. In: WORK- 
SHOP ON PLANT ADAPTATION TO MINERAL STRESS IN PROBLEM SOILS, Beltsville, Maryland, 1976, edited by Madison J. Wright. Proceedings. Ithaca, Cornell University, 1976.

CLARKSON, D.T. \& SANDERSON, J. Inhibition of uptake and long distance transport of calcium by aluminum and other polyvalent cations. Journal Experimental Botany, 22:837-851, 1971.

KERRIDGE, P.C. Aluminum toxicity in wheat (Triticum aestivum Vill., Host). Corvallis, Oregon State University, 1969. 170f. Tese (Doutoramento)

MOORE, D.P.; KRONSTAD, W.E. \& METZGER, R. Screening wheat for aluminum tolerance. In: WORKSHOP ON PLANT ADAPTATIONS TO MINERAL STRESS IN PROBLEM SOILS, Beltsville, Maryland, 1976, edited by Madison J. Wright. Proceedings. Ithaca, Cornell University, 1976. p. 287-295.

OUELLETTE, G.J. \& DESSUREAUX, L. Chemical composition of alfalfa as related to degree of tolerance to manganese and aluminum. Canadian Journal of Plant Science, 38:206-214, 1958. 